\title{
Incipient Smoke Residues it's Effect and Prevention: A Review
}

\author{
Rumana Perveen ${ }^{1}$, Rahul Srivastava ${ }^{2}$, Rohit Kumar ${ }^{3}$, Saddab Alam ${ }^{4}$ \\ ${ }^{1}$ Consultant, Dental Surgeon Ahead Academy, New Delhi, ${ }^{2}$ Reader, Department of Oral Medicine and Radiology, Rama Dental \\ College Hospital and Research Centre, Kanpur, ${ }^{3}$ Consultant, Dental Surgeon Gopalganj, Bihar, ${ }^{4}$ Consultant, Dental Surgeon \\ Gopalganj, Bihar, India
}

Corresponding author: Dr. Rumana Perween, 57/11 Old Rajendra Nagar, New Delhi-110060, India

DOI: http://dx.doi.org/10.21276/ijcmsr.2019.4.3.24

How to cite this article: Rumana Perveen, Rahul Srivastava, Rohit Kumar, Saddab Alam. Incipient smoke residues it's effect and prevention: a review. International Journal of Contemporary Medicine Surgery and Radiology. 2019;4(3):C106-C109.

\section{A B S T R A C T}

Thirdhand smoke (THS) is a film of invisible toxic particles leftover from cigarette smoke. It can stick to almost any surface, and once it does, those noxious layers build up and can stay there for months. Thirdhand smoke (THS) has a far reach sticking to the floors, rugs, carpets, curtains and walls of most rooms. Thirdhand smoke (THS) contains more than 250 chemicals. Thirdhand smoke (THS) and is increasingly recognized as a potential danger, especially to children, who not only inhale fumes released by these residues but also ingest residues that get on their hands after crawling on floors or touching walls and furniture. This review highlights the characteristics, route of exposure, health effect and prevention of thirdhand smoke.

Keywords: Nicotine, Thirdhand Smoke, Smoking, Cigarette

\section{INTRODUCTION}

Mainstream smoke (MSS), or first hand smoke, is created by tobacco combustion at $600-900^{\circ} \mathrm{C}$, when smokers inhale the smoke from a burning cigarette, whereas side stream smoke (SSS) emanates from the smoldering end of a lit cigarette at approximately $600^{\circ} \mathrm{C}$ between puffs. Secondhand smoke (SHS), or environmental smoke, is a mixture of SSS (approx.85\%) and exhaled MSS (approx.15\%). ${ }^{1}$

The toxicity of low levels of constituents of tobacco smoke has been demonstrated. According to the National Toxicology Program these 250 poisonous gases, chemicals, and metals include hydrogen cyanide (used in chemical weapons), carbon monoxide (found in car exhaust), butane (used in lighter fluid), ammonia (used in household cleaners), toluene (found in paint thinners), arsenic (used in pesticides), lead (formerly found in paint), chromium (used to make steel), cadmium (used to make batteries), and polonium-210 (highly radioactive carcinogen). Eleven of these compounds are group 1 carcinogens (most carcinogenic designation). The word "thirdhand smoke" was first invented by scientists at the Massachusetts General Hospital in Boston, MA in 2006 with the Clinical Effort Against Second Hand Smoke Exposure (CEASE) program and latest study has started to shed significant light on the subject. In 2011, a study consortium of researchers financed by the California Tobacco-Related Disease Research Program was established to carry out groundbreaking research into the characterization, exposure and health impacts of third hand tobacco smoke., ${ }^{2,3}$

Thirdhand smoke (THS) is defined as the contamination of surfaces in contact with compounds emitted in SHS, the novel products generated by chemical transformations of the components, and the off-gassing of volatile components into the air. The phrase "the four Rs" provides a working definition of THS: tobacco chemicals that remain, react, re-emit, and/ or are resuspended long after active smoking has ceased. ${ }^{1,3,4}$ When tobacco is smoked, the particles in secondhand smoke settle on surfaces; this contamination is deeply absorbed into materials such as hair, clothing, carpeting, furniture and wallboard. The chemicals undergo an aging process after depositing on surfaces, which changes the chemical structure of the smoke pollutants. The nicotine in third hand smoke residue reacts with common indoor air pollutants, such as nitrous acid and ozone, to form hazardous substances. As third hand smoke ages, it becomes more toxic. ${ }^{5}$

Is third-hand smoke different from second-hand smoke?

1. Second-hand smoke is a mixture of chemicals that are produced by burning tobacco and the smoke that the person smoking exhales. Second-hand smoke can cause disease and death just like primary smoking.

2. Third-hand smoke persists long after putout of the cigarette. For months toxins can stay in homes, long after people who have smoked move out.

\section{Characteristics of Third Hand Smoke}

Third-hand smoke consists of toxins remaining after putout of the cigarette, cigar or pipe. It will combine with household dust and other particles, building up and becoming more toxic overtime. Third-hand smoke sticks to items such as:

1. Carpeting.

2. Flooring.

3. Furniture.

4. Walls.

5. Clothing. 
6. Toys.

7. Hair.

8. Skin.

9. Vehicle surfaces. ${ }^{6}$

Dust, air and surfaces of indoor contain tobaccorelated compounds such as nicotine, polycyclic aromatic hydrocarbons (PAHs), volatile N-nitrosamines, and tobaccospecific nitrosamines (TSNAs). Substances such as TSNAs including NNK (4-(methylnitrosamino)-1-(3-pyridyl)1-butanone), NNN (N-nitrosonornicotine) and NNA (1-(N-methyl-N-nitrosamino)-1-(3-pyridinyl)-4-butanal), cotinine and formaldehyde are formed when nicotine reacts with ambient gases present in the indoor environment (including ozone and nitrous acid). THS has significant longevity and some gas and particle phase. THS compounds can remain in indoor environments for days to months after smoking has last taken place. THS residues that are deposited on surfaces can be resuspended back into the air over time, providing a lingering source of tobacco-related compounds. THS release from, or through, surfaces (desorption) is affected by the relative humidity and air exchange rate of the indoor environment and varies by surface type..$^{7,8}$

Matt GE et al examined pollutants from tobacco smoke (also known as THS) that remained in former smokers ' homes and exposure to these pollutants.

Measures were repeated 1 week (W1), 1 month (M1), 3 months (M3) and 6 months (M6) after cessation among verified quitters. Measures for THS pollutants on household surfaces, fingers and dust (i.e., nicotine, tobacco-specific nitrosamines) and urinary exposure markers (i.e. cotinine, 4-(methylnitrosamine)-1-(3-pyridyl)-1-butanol (NNAL) were analyzed. He concluded that Smoker's home remained contaminated with THS for up to 6 months following cessation. Before cessation of smoking, residents continued to be exposed to THS toxicants accumulating in settled house dust and surfaces. ${ }^{9}$

\section{Routes of exposure}

THS exposure can occur through:

1. Involuntary ingestion: Ingestion occurs through household objects that are contaminated with tobacco smoke residue in mouth (eating utensils, toothbrushes).

2. Dermal (skin) absorption: Dermal absorption occurs when individual touch a surface on which THS has accumulated, because it can be absorbed through skin.

3. Inhalation: Inhale THS in the air. ${ }^{7,10}$

\section{Unintentional Exposure}

1. THS found in the homes of non-smokers and in homes where smoking is not currently allowed, but where smokers have previously lived.

2. THS was discovered in both smoking and non-smoking rooms in hotels with only partial smoking restrictions.

3. Rental cars and used cars may be contaminated with THS depending on smoking rates and vehicle turnover frequency.

4. Used cars that were permitted to smoke or sold by smokers who had car smoking bans were shown to be contaminated with THS at the time of sale.

5. Homes of smokers who quit long after inhabitants stopped smoking discovered THS contamination. High levels of nicotine and cancer causing tobacco chemicals in house dust were found six months after quitting smoking in former smoker's homes. ${ }^{11}$

Matt GE et al conducted a study to investigate whether dust and surfaces in smoker's households are contaminated with environmental tobacco smoke (ETS), whether smoking parents can protect their children by smoking outside and away from the infant, and whether contaminated dust, surfaces and air contribute to ETS in infants. In household dust, indoor air, and household surfaces, ETS contamination was measured by nicotine. Exposure to ETS was evaluated in infant urine by cotinine levels. It was found that ETS contaminates dust and surfaces in smoker's homes. Smoker's infants are at danger of exposure to ETS through dust, surfaces, and air in their homes. Smoking outside the home and away from the child decreases but does not safeguard the home of a smoker from contamination with ETS and the infant of a smoker from exposure to ETS. ${ }^{12}$

In 2008, Matt GE et al detected nicotine in dust (mean 19.51 $\mu \mathrm{g} / \mathrm{g}$ ) and dashboards (mean $8.61 \mu \mathrm{g} / \mathrm{m}^{2}$ ) of 78 vehicles belonging to people who smoked in their vehicles compared to dust (mean $3.37 \mu \mathrm{g} / \mathrm{g}$ ) and dashboards (mean $0.06 \mu \mathrm{g}$ $/ \mathrm{m}^{2}$ ) of 20 non-smoker vehicles. For at least 12 months, eight smokers had implemented a smoking ban on their cars. However, their vehicles were contaminated with nicotine (mean dust of $11.61 \mu \mathrm{g} / \mathrm{g}$ and dashboard of $5.09 \mu \mathrm{g} / \mathrm{m}^{2}$ ). ${ }^{13}$

\section{Who supposed to be exposed??}

\section{Children and families}

A. It is more probable that spouses, kids and others who come into close contact with smokers will be subjected to THS.

B. Babies, toddlers, and children are at greater risk of negative health effects because:

- They inhale 40 times more than adults.

- They have greater hand/object/mouth contact so they absorb proportionately more through ingestion have greater absorption through their skin.

C. Children's growing bodies make them more vulnerable to THS than adults.

D. Even when parents and caregivers only smoke outdoors, children are not fully protected from exposure to tobacco because THS stays on smokers ' clothes, skin, and hair, and smoke can drift indoors. ${ }^{11,14}$

\section{Rental housing}

People living in rental homes or flats are at a greater danger of exposure to THS because these properties commonly alter occupants, and smoking bans often do not apply to personal areas like homes. Children living in flats have a greater amount of tobacco chemicals exposure than kids living in detached accommodation, even if they are not subjected to secondhand smoke. These kids may be subjected to THS from prior tenants who smoked at home or from neighborhood smoke that enter their home through open doors, air ducts, or other paths. ${ }^{11}$

Matt GE et al performed a survey to investigate whether third-hand smoke (THS) persists in smoker's households after moving out and non-smokers move in, and whether 
fresh non-smokers in these households are subjected to THS. He found that THS accumulates in smokers ' homes and persists even after houses stay empty for two months when smokers move out and are cleaned and ready for fresh inhabitants. When nonsmokers move into homes formerly occupied by smokers, they encounter indoor environments with THS polluted surfaces and dust. ${ }^{15}$

\section{Occupational exposure}

Employees and clients in settings where smoking is permitted (such as hotels, casinos or long-term indoor smoking facilities) are more likely to be exposed to thirdhand smoke. ${ }^{11}$

\section{Health hazards of thirdhand smoke (THS)}

\section{Lung cancer}

Studies indicated that third hand smoke exposure induced DNA double-strand breaks and increased cell proliferation and colony formation. In addition, RNA sequencing analysis revealed that third hand smoke exposure caused endoplasmic reticulum stress and activated p53 (tumor suppressor) signaling. Physiological, cellular and molecular information show that an enhanced risk of lung cancer is associated with early exposure to third-hand smoke. ${ }^{16}$

\section{Damage to human DNA}

Hang $\mathrm{B}$ et al conducted a study to assess the genotoxicity of THS in human cell lines using two in vitro assays. THS was generated in laboratory systems that simulated short (acute)and long (chronic)-term exposures. The long amplicon -quantitative PCR assay quantified significantly higher levels of oxidative DNA damage in hypoxanthine phosphor ribosyl transferase 1 (HPRT) and polymerase $\beta$ (POLB) genes of cultured human cells exposed to chronic THS for $24 \mathrm{~h}$ compared with untreated cells, suggesting that THS exposure is related to increased oxidative stress and could be an important contributing factor in THS-mediated toxicity. The results from this study demonstrated that exposure to THS is genotoxic in human cell lines. ${ }^{17}$

\section{Formation of carcinogens by Airborne chemicals}

An important feature of THS is its ability to undergo chemical transformations during aging periods, as shown in a recent study that sorbed nicotine reacts with indoor pollutant nitrous acid (HONO) to form tobacco-specific nitrosamines (TSNAs) such as 4-(methylnitrosamine)-4-(3pyridyl)butanine (NNA) and 4-(methylnitrosamine)-1-(3pyridyl)-1-butanone (NNNK). ${ }^{17}$

\section{Effect on children}

Tobacco particulates have been associated with cognitive deficits among children. The greater level of exposure to tobacco, the greater risk of deficits in reading. Studies have shown the link between third-hand smoke and infant pulmonary and respiratory diseases as well as increased opportunities for asthma and otitis media (infection of the middle ear) and poor physique. Significantly greater quantities of urine cotinine, a biomarker for nicotine exposure, have been identified among children living in households where smoking occurs inside compared to homes where smokers go outside to smoke. Mahabee-Gittens EM has found a positive correlation between the amount of nicotine found on the hands of children and the amount of cotinine, a biomarker for nicotine exposure, detected in the saliva of children. ${ }^{12,18,19}$

\section{Prevention}

1. Traditional techniques of cleaning may not properly remove nicotine that adsorbs to indoor surfaces due in part to the capacity of nicotine to permeate all components of enclosed areas, such as dust and air, porous construction materials (e.g. sheetrock and drywall), doors, cabinets, curtains, furniture / upholstery, bedding / pillows / mattresses, clothing supplies and carpets. $^{20}$

2. An outdoor waiting period of 10 minutes after a cigarette before entering a building can decrease these types of indoor air pollution.

3. Avoid smoking around kids. Choose a location outside to smoke, away from where kids play. Ask other adults outside to smoke. Opening windows does not provide sufficient protection against second and third-hand smoke.

4. Avoid smoking around children. Choose a place to smoke outside, away from where children play. Ask other adults to smoke outside. Opening windows is not enough protection against second and thirdhand smoke.

5. Ask visitors, babysitters, grandparents and friends not to smoke in home or around children. Inform them that the smoke can increase child's risk for developing health problems (asthma, bronchitis, pneumonia, and middle ear problems).

6. Do not allow smoking in home or car, even when children are not present.

7. Choose a smoke-free day care center.

8. Visit places where smoking is prohibited.

\section{For help with quitting}

1. Talk to health care provider to find out if person is eligible to use medications or nicotine replacement products to help quit smoking.

2. Find a local smoking cessation program by visiting http://www.quitnet.com/ library/programs/.

3. Call 1-800-QUITNOW (1-800-7848669) for telephone counseling and information about quitting in state.

4. Don't give up! Keep trying. ${ }^{21}$

\section{CONCLUSION}

THS residues are present in the air, dust, and on surfaces of multiple indoor environments where smoking has previously occurred, including homes and cars. These residues contain a variety of tobacco-related compounds. Researchers are still studying its possible dangers. In the meantime, the only way to protect nonsmokers from THS is to create a smoke-free environment.

\section{REFERENCES}

1. Hang B,Wang P, Zhao Y, Sarker A, Chenna A, Xia Y,Antoine M. Snijders AM, Mao JH. Adverse Health Effects of Thirdhand Smoke: From Cell to Animal Models. Int. J. Mol. Sci. 2017;18(1):1-8.

2. Winickoff JP, Friebely J, Tanski SE, Sherrod C,Matt GE,Hovell MF, McMillen RC. Beliefs About the 
Health Effects of "Thirdhand" Smoke and Home Smoking Bans Pediatrics. 2009;123 (3):74-79.

3. Matt et al. Third hand tobacco smoke: emerging evidence and arguments for a multidisciplinary research agenda. Environ Health Perspect. 2011;119 (5):1218-26.

4. Jacob P.; Benowitz, N.L.; Destaillats, H.; Gundel L.; Hang, B.; Martins-Green, M.; Matt, G.E.; Quintana, P.J.; Samet, J.M.; Schick, S.F.; et al. Thirdhand Smoke: New Evidence, Challenges, and Future Directions. Chem. Res. Toxicol. 2017; 30 (2): 270-294.

5. Drehmer JE, Walters BH, Burza EN, Winickoff JP. Guidance for the Clinical Management of Thirdhand Smoke Exposure in the Child Health Care Setting J Clin Outcomes Manag. 2017;24 (4):551-559.

6. Third Hand Smoke - Health Diary. Available from: ? FName=/Client_PDFs/2541.pdf

7. Third-hand tobacco smoke exposure and implications for public health A background paper. Available from: https://www.cph.co.nz/wp-content/uploads/ thsbackgroundpaper.pdf

8. Burton A Does the Smoke Ever Really Clear? Thirdhand Smoke Exposure Raises New Concerns. Environ Health Perspect. 2011;119 (3): 70- 74.

9. Matt GE, Quintana PJE, Zakarian JM et al When smokers quit: exposure to nicotine and carcinogens persists from thirdhand smoke pollution Tobacco Control 2017;26 (6):548-556.

10. Eldridge L, Third-Hand Smoke Exposure Routes, Risks, and Dangers. Available from: https:// www.verywellhealth.com/what-is-third-handsmoke-2248867.

11. Thirdhand Smoke Fact Sheet - California Department of Public Health Available from https://www.cdph.ca.gov/ Programs/ CCDPHP/.../ThirdhandSmokeFactSheet. pdf.

12. Matt GE, Quintana PJ, Hovell MF, et al. Households contaminated by environmental tobacco smoke: sources of infant exposures. Tob Control. 2004; 13 (3):29-37.

13. Matt GE, Quintana PJ, Hovell MF, Chatfield D, Ma DS, Romero R, Uribe A. Residual tobacco smoke pollution in used cars for sale: air, dust, and surfaces. Nicotine Tob Res. 2008;10 (6):1467-75.

14. Third Hand Smoke, The Hidden Hazard of Third Hand Smoke. Available from: https://www.neha.org/ehtopics/air-quality-0/third-hand-smoke.

15. Matt GE, Quintana PJE, Zakarian JM, Fortmann AL,Chatfield DA, Hoh E, Uribe AM, Hovell MF When smokers move out and nonsmokers move in: Residential thirdhand smoke pollution and exposure. Tob Control. 2011;20 (1):1-18.

16. Third-hand smoke found to increase lung cancer risk in mice. Available from: https://www.sciencedaily.com / releases/ 2018/03 / 180309095539.htm

17. Hang B, Sarker AH, Havel C, Saha S, Hazra TK, Schick. Thirdhand smoke causes DNA damage in human cells. Mutagenesis 2013;28 (5):381-391.

18. Mahabee-Gittens EM, Merianos AL, Matt GE. Preliminary evidence that high levels of nicotine on children's hands may contribute to overall tobacco smoke exposure. Tob Control. 2018;27 (3):217-219.

19. Risks Third-hand Smoking. Available from: https://www.
smokefree.hk/en/content/web.do?page=ThirdhandSm oking.

20. Northrup TF, Jacob P, Benowitz NL, Hoh E, Quintana PJE et al Thirdhand Smoke: State of the Science and a Call for Policy Expansion Public Health Rep. 2016;131 (6): 233-238.

21. What is Second and Third-hand Smoke? - American Thoracic Society. Available from: https://www.thoracic. org/patients/patient-resources/resources/second-handsmoke.pdf.

\section{Source of Support: Nil; Conflict of Interest: None}

Submitted: 27-06-2019; Accepted: 22-07-2019; Published online: 19-08-2019 